\title{
Reductively degradable polyester-based block copolymers prepared by facile polycondensation and ATRP: synthesis, degradation, and aqueous micellization $\dagger$
}

\author{
Andrew Nelson-Mendez, $\ddagger^{a}$ Samuel Aleksanian, $\ddagger^{a}$ Misook Oh, ${ }^{b}$ Hyun-Suk Lim ${ }^{b}$ and Jung Kwon Oh*a \\ Received 29th April 2011, Accepted 6th June 2011 \\ DOI: 10.1039/c1sm05781b
}

\begin{abstract}
Well-defined reductively degradable amphiphilic block copolymers having disulfide linkages positioned repeatedly on hydrophobic chains, thus exhibiting fast degradation, were prepared by a combination of polycondensation and ATRP. The new method consists of three synthetic steps including, (1) polycondensation of commercially available diols and diacids through carbodiimide coupling or high temperature processes to synthesize degradable polyesters with disulfides labeled on the main chain at regular intervals (ssPES-OH), (2) bromination of ssPES-OH to ssPES-Br, and (3) ATRP for chain extension of ssPES-Br with water-soluble polymethacrylate, yielding ssPES- $b$-polymethacrylate block copolymers (ssABPs). The reductive cleavage of disulfide linkages in reducing conditions resulted in the degradation of ssPES homopolymers; their degradation rate was significantly enhanced with the increasing amounts of disulfide linkages in ssPES-OH and reducing agents. For ATRP, gel permeation chromatography and ${ }^{1} \mathrm{H}-\mathrm{NMR}$ results confirmed the synthesis of well-defined ssABPs and revealed that polymerizations were well controlled. Because of their amphiphilic nature, ssABPs self-assembled in water toward the formation of core/shell micelles consisting of a hydrophobic ssPES core surrounded with polymethacrylate coronas. The effects of the corona's chain length on thermal properties and micellization in water of well-defined ssABPs were examined. Moreover, reductive (or thiol-responsive) degradation of ssABP-based micelles enabled fast release of encapsulated model drugs. Cell culture experiments confirmed nontoxicity and biocompatibility of well-defined ssABPs as effect candidates for targeted delivery applications.
\end{abstract}

\section{Introduction}

Well-defined amphiphilic block copolymers (ABPs) have great potential as building blocks to construct nanostructured materials in biomedicine, pharmaceutics, cosmetics, and bionanotechnology. ${ }^{1} \mathrm{ABPs}$ self-assemble in aqueous solutions to form core/shell micelles consisting of a hydrophobic core, capable of carrying a variety of bioactive molecules including therapeutics, surrounded with hydrophilic coronas, to ensure water-solubility and biocompatibility. ${ }^{2}$ These micelles offer many advantages as polymer-based drug delivery systems (DDS). ${ }^{3}$ Their advantageous properties include facile preparation, colloidal stability with low critical micellar concentration (CMC), and tunable sizes with narrow size distribution, as well as high physical loading

${ }^{a}$ Department of Chemistry and Biochemistry, Concordia University, 7141 Sherbrooke St W., Montreal, Quebec, Canada H4B 1R6. E-mail: joh@, alcor.concordia.ca

${ }^{b}$ Department of Biochemistry and Molecular Biology, Indiana University School of Medicine, Indianapolis, Indiana, 46202, USA

$\dagger$ Electronic supplementary information (ESI) available. See DOI: $10.1039 / \mathrm{c} 1 \mathrm{sm} 05781 \mathrm{~b}$

\$ Authors equally contributed. efficiency of drugs without chemical modification and improved pharmacokinetics. They can also protect drugs from possible deactivation and preservation of their activities during circulation, transportation to targeted organ or tissue, and intercellular trafficking. However, the present and future design and development still require a high degree of control over properties. ${ }^{4,5}$

Fast and controllable release of encapsulated drugs is greatly desired in the development of ABP-based micellar DDS. ${ }^{6-8}$ In the recent years, much effort has been directed toward the development of stimuli-responsive degradable micelles. In general, these micelles contain cleavable linkages that can degrade in response to external stimuli, causing the micelles to dissociate. The typical external triggers include low $\mathrm{pH}$ or light, as well as reductive, oxidative, or enzymatic reactions. ${ }^{9}$ Reductive reaction utilizes reductively degradable disulfide linkages. Disulfides are cleaved into their corresponding thiols in two ways: under reducing environments in the presence of reducing agents such as tributyl phosphine $\left(\mathrm{Bu}_{3} \mathrm{P}\right)^{10,11}$ and through a disulfide-thiol exchange in the presence of water-soluble thiols such as dithioerythritol (DTT) ${ }^{12-14}$ and glutathione (GSH, a tripeptide containing cysteine with a pendent thiol group). ${ }^{15}$ In biological environments, GSH is found in animal cells at millimolar 
concentrations, enabling biodegradation. ${ }^{16}$ Several approaches to the disruption of micelles have been proposed. One approach involves the introduction of cleavable crosslinkers functionalized with disulfide ${ }^{13,17}$ or acid-labile acetal ${ }^{18,19}$ during micellization or post-micellization to yield crosslinked micelles. ${ }^{20}$ Another approach involves the incorporation of a cleavable group at the junction of hydrophilic and hydrophobic blocks. Typical examples include the synthesis of sheddable micelles containing mainly photo-cleavable, ${ }^{21-23}$ disulfide,${ }^{24}$ and recently hydrazone ${ }^{25}$ linkages. The main established approach is to incorporate stimuli-responsive cleavable (or degradable) groups onto the hydrophobic block of ABPs. The cleavable groups are positioned as pendent groups of the hydrophobic main chain. ${ }^{26-31}$ However, the method is limited in that it only works when specific monomers are synthesized. The promising method is to develop degradable block copolymers containing cleavable groups in the hydrophobic block positioned repeatedly along the hydrophobic main chain. ${ }^{32}$

Controlled radical polymerization ${ }^{33,34}$ has proven to be useful for the construction of well-controlled ABPs as effective delivery carriers. In addition to reversible addition-fragmentation chain transfer (RAFT) polymerization ${ }^{35-37}$ and nitroxide-mediated radical polymerization (NMP), ${ }^{38,39}$ atom transfer radical polymerization (ATRP) has been extensively utilized for the preparation of well-controlled ABPs,${ }^{40-43}$ particularly polyester-based ABPs. ${ }^{44-46}$

Herein we report the synthesis of reductively degradable (or thiol-responsive) ABPs with disulfide linkages positioned repeatedly on the main chain at regular intervals, allowing for fast reductive degradation and thus rapid and controlled release of encapsulated model drugs. These well-defined ABPs were prepared by the combined polycondensation and ATRP. This new method includes three synthetic steps (Scheme 1). The first step is the polycondensation of commercially available diols and diacids to prepare degradable polyesters labeled repeatedly with disulfide linkages (ssPES-OH) on the main chain. A series of ssPES-OH containing different diols reacting with 3,3'-dithiodipropionic acid (SS-Dacid) was synthesized by polycondensation through carbodiimide coupling or high temperature processes. The effect of the amounts of disulfide linkages in ssPES-OH and reducing agents on degradation of these ssPES-OH homopolymers was examined. The second step is the bromination of ssPES-OH to prepare ssPES- $\mathrm{Br}$ as an ATRP macroinitiator. The third step involves ATRP for chain extension of ssPES-Br with water-soluble polymethacrylate, allowing for the synthesis of disulfide-labeled degradable ssABPs. Well-defined ssABPs having different chain lengths of polymethacrylate block were further characterized with thermal analysis and micellization in water. The applicability for biological applications of these degradable ssABPs as drug delivery vehicles was initially assessed with cell viability measurements.

\section{Experimental}

\section{Materials}

2-Hydroxyethyl disulfide (SS-DOH), 1,12-dodecyl diol (C12-DOH), 3,3'-dithiodipropionic acid (SS-Dacid), N, $N^{\prime}$-dicyclohexyl carbodiimide (DCC), 4-dimethylaminopyridine (DMAP), triethylamine $\left(\mathrm{Et}_{3} \mathrm{~N}\right), \alpha$-bromoisobutyryl bromide (Br-iBuBr), 1,1,4,7,10,10-hexamethyltriethylenetetramine (HMTETA, 97\%), 2,2'-bipyridyl (bpy), $N, N, N^{\prime}, N^{\prime \prime}, N^{\prime \prime}$-pentamethyldiethylenetriamine (PMDETA, >98.0\%), copper(I) bromide (CuBr, >99.99\%), copper(II) bromide $\left(\mathrm{CuBr}_{2},>99.99 \%\right), 0.102 \mathrm{~N}$ $\mathrm{KOH}$ standard solution in methanol, tributyl phosphine $\left(\mathrm{Bu}_{3} \mathrm{P}\right.$, 97\%), $p$-toluene sulfonic acid ( $p$-TSA), Nile Red (NR), and anisole from Aldrich were used as received. DL-Dithioerythritol (DTT, 99\%) was purchased from Acros Organics. Oligo(ethylene glycol) monomethyl ether methacrylate (OEOMA) with $M=$ $300 \mathrm{~g} \mathrm{~mol}^{-1}$ and pendent EO units DP $\approx 5$ from Aldrich was purified by passing it through a column filled with basic alumina to remove inhibitors.

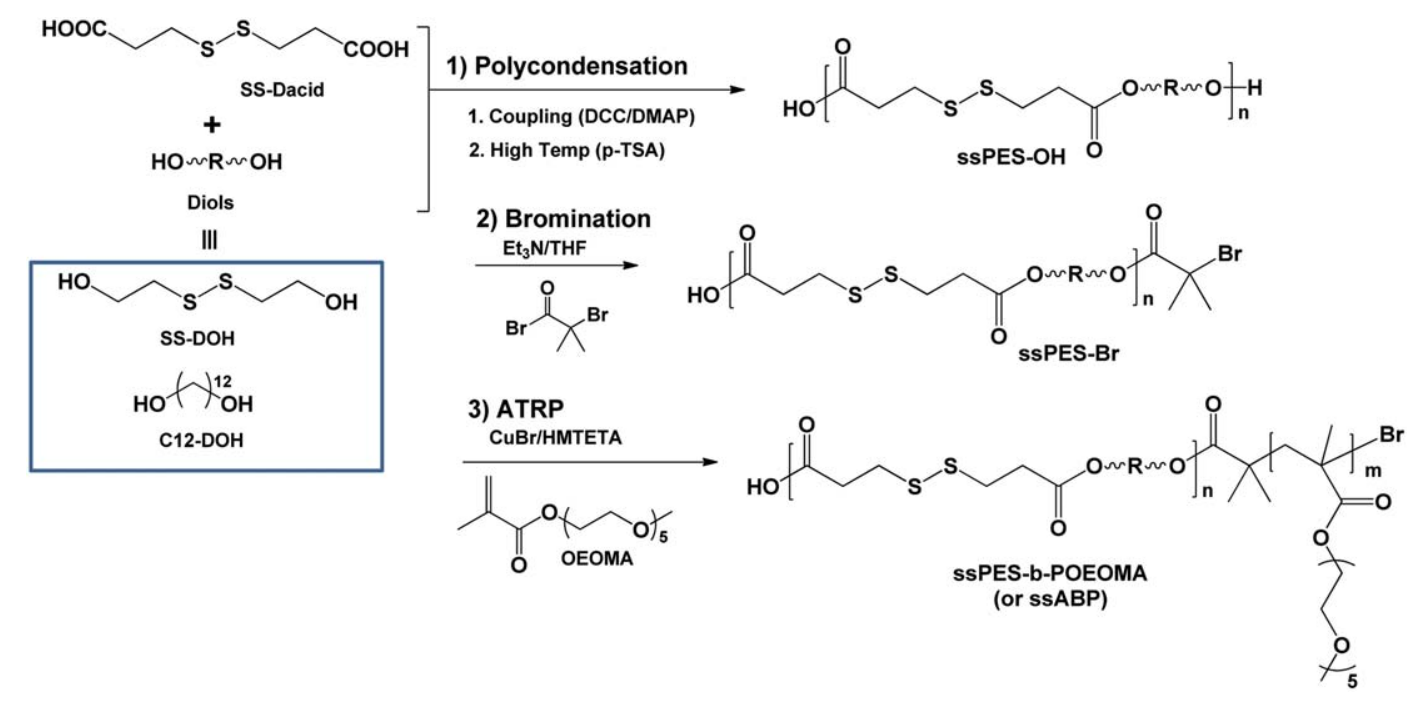

Scheme 1 Synthetic route for reductively degradable ssABPs consisting of ssPES block labeled repeatedly with disulfide linkages along hydrophobic main chains and a water-soluble POEOMA block. 


\section{Polycondensation for synthesis of ssPES-OH}

Carbodiimide coupling of SS-Dacid and different diols (SS-DOH and $\mathrm{C} 12-\mathrm{DOH})$ in the presence of DCC was examined to synthesize a series of ssPES-OH. The procedure for the synthesis of ssPES-2 containing SS-DOH is as follows: SS-Dacid (26.8 g, $0.127 \mathrm{~mol}$ ) dissolved in tetrahydrofuran (THF, $100 \mathrm{~mL}$ ) was drop-wise added to a solution of SS-DOH (20 g, $0.130 \mathrm{~mol})$, DCC (53.6 g, $0.260 \mathrm{~mol})$, a catalytic amount of DMAP, and THF $(200 \mathrm{~mL})$ at $0{ }^{\circ} \mathrm{C}$ over $30 \mathrm{~min}$. The resulting solution was stirred at room temperature overnight. Formed white solids were removed by a vacuum filtration, and then the solvent was removed by rotary evaporation. An aliquot of the product (5 g) was precipitated from $\mathrm{MeOH}(50 \mathrm{~mL})$ to remove $\mathrm{MeOH}$-soluble, low molecular weight species. The precipitated ssPES-2 was dried in a vacuum oven at room temperature overnight. Yield $=$ $30.4 \mathrm{~g}(65 \%)$.

Similar procedure was utilized for the synthesis of ssPES-4, except for the use of SS-Dacid $(6.0 \mathrm{~g}, 0.029 \mathrm{~mol}), \mathrm{C} 12-\mathrm{DOH}$ $(6.2 \mathrm{~g}, 0.031 \mathrm{~mol}), \mathrm{DCC}(6.4 \mathrm{~g}, 0.031 \mathrm{~mol})$, a catalytic amount of DMAP, and THF (150 mL). The product was purified by precipitation from $\mathrm{MeOH}$. Yield = $6.5 \mathrm{~g}(53 \%)$.

High temperature polycondensation of SS-Dacid and C12$\mathrm{DOH}$ in the presence of $p$-TSA, a Lewis catalyst, was examined to synthesize relatively high molecular weight ssPES-OH homopolymers. The typical procedure for the synthesis of ssPES6 containing $\mathrm{C} 12-\mathrm{DOH}$ is as follows: SS-Dacid (1.04 g, $4.9 \mathrm{mmol}), \mathrm{C} 12-\mathrm{DOH}(1.0 \mathrm{~g}, 4.9 \mathrm{mmol})$, and $p$-TSA $(0.08 \mathrm{~g}$, $0.4 \mathrm{mmol}$ ) were charged and the mixture was magnetically stirred at $180-185^{\circ} \mathrm{C}$ for a given time. The dark brown melt was cooled at room temperature, dissolved in THF, and then precipitated from $\mathrm{MeOH}(200 \mathrm{~mL})$. The precipitated ssPES-6 was dried in a vacuum oven at room temperature overnight. Yield $=0.8 \mathrm{~g}(41 \%)$.

\section{Bromination of ssPES-OH to ssPES-Br}

A solution of $\mathrm{Br}-\mathrm{BBuBr}(9.43 \mathrm{~g}, 41.2 \mathrm{mmol})$ in THF $(20 \mathrm{~mL})$ was added drop-wise to a solution of ssPES-2 (33.8 g, $19.3 \mathrm{mmol})$ in a mixture of $\mathrm{Et}_{3} \mathrm{~N}(5.8 \mathrm{~mL}, 41.1 \mathrm{mmol})$ and THF $(280 \mathrm{~mL})$. The resulting dispersion was stirred overnight. Formed white solids were removed by vacuum filtration and the solvent was removed by rotary evaporation. Crude product was re-dissolved in $\mathrm{MeCl}_{2}$ and the mixture was washed with an aqueous $\mathrm{NaHCO}_{3}$ solution and an aqueous $\mathrm{HCl}$ solution (2 times each). The solvent was removed by rotary evaporation. The residue was precipitated from $\mathrm{MeOH}$ to isolate ssPES-Br. Yield $=18.7 \mathrm{~g}(55 \%)$.

\section{ATRP for synthesis of ssPES- $b$-POEOMA block copolymers (ssABPs)}

A series of ATRP of OEOMA was conducted in the presence of the ssPES-Br macroinitiator in anisole at $47^{\circ} \mathrm{C}$ under different conditions. The detailed procedure for ATRP-2 is as follows: dried, purified ssPES-2-Br (0.5 g, $0.33 \mathrm{mmol})$, OEOMA (5.6 g, $18.7 \mathrm{mmol})$, HMTETA ( $45 \mu \mathrm{L}, 0.167 \mathrm{mmol})$, and anisole $(6 \mathrm{~mL})$ were added to a $25 \mathrm{~mL}$ Schlenk flask. The resulting mixture was deoxygenated by three freeze-pump-thaw cycles. The reaction flask was filled with nitrogen and then $\mathrm{CuBr}(23.9 \mathrm{mg}$, $0.167 \mathrm{mmol}$ ) was added to the frozen solution. The flask was closed, evacuated with vacuum and backfilled with nitrogen three times. The mixture was thawed and an initial sample was taken via a syringe. The flask was then immersed in an oil bath preheated to $47{ }^{\circ} \mathrm{C}$ to start the polymerization. Aliquots were withdrawn at different time intervals during the polymerization to monitor conversion and molecular weight by GPC. The polymerization was stopped by exposing the reaction mixture to air.

The resulting ssABPs were purified by removal of residual copper species and unreacted monomers. As-prepared green polymer solutions $(5 \mathrm{~g})$ were drop-wise added into hexane $(150 \mathrm{~mL})$ under stirring to precipitate green residues. The precipitated polymers were passed through a column filled with basic aluminium oxide with THF as an eluent to remove copper species. The solvent was removed by rotary evaporation and residual solvents were further removed in a vacuum oven at $50^{\circ} \mathrm{C}$ overnight, resulting in dried, purified ssABP.

\section{Instrumentation and analyses}

${ }^{1} \mathrm{H}-\mathrm{NMR}$ spectra were recorded using a $500 \mathrm{MHz}$ Varian spectrometer with $\mathrm{CDCl}_{3}$ singlet at $7.29 \mathrm{ppm}$ and DMSO-d 6 at $2.5 \mathrm{ppm}$ as reference standards. Molecular weight and molecular weight distribution were determined by gel permeation chromatography (GPC) with a Viscotek VE1122 pump and a refractive index (RI) detector. Three PolyAnalytik columns (PAS$103 \mathrm{~L}, 105 \mathrm{~L}, 106 \mathrm{~L}$, designed to determine molecular weight up to $2000000 \mathrm{~g} \mathrm{~mol}^{-1}$ ) were used with THF as an eluent at $30^{\circ} \mathrm{C}$ at

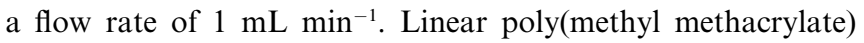
(PMMA) standards were used for calibration. Aliquots of polymer samples were dissolved in THF and the clear solutions were filtered using a $0.25 \mu \mathrm{m}$ PTFE filter to remove any THFinsoluble species. A drop of anisole was added as a flow rate marker. Conversion was also determined using GPC by following the decrease in the macromonomer (OEOMA) peak area relative to the increase in the polymer peak area. The particle size was measured by dynamic light scattering (DLS) on a Particle Sizer, Model DynaPro Titan from Wyatt Technology. The critical micellar concentration (CMC) of block copolymers in water was determined on a DeltaPi Surface Tensiometer from Kibron at room temperature. Thermal properties including glass transition temperature $\left(T_{\mathrm{g}}\right)$ and melting temperature $\left(T_{\mathrm{m}}\right)$ of polymers were measured with a TA Instruments DSC Q10 differential scanning calorimeter over a temperature range of -70 to $150{ }^{\circ} \mathrm{C}$ at a heating rate of $10^{\circ} \mathrm{C} \mathrm{min}^{-1}$.

\section{Characterization}

The concentration of terminal $\mathrm{COOH}$ groups in ssPES-OH was determined using an acid-base titration. Typically, the dried, purified ssPES-3 (503.6 mg, $2.74 \mathrm{mmol}$ ) was dissolved in a mixture of $1 / 1 \mathrm{v} / \mathrm{v} \mathrm{THF} / \mathrm{MeOH}(150 \mathrm{~mL})$ containing phenolphthalein as a $\mathrm{pH}$ indicator. $0.102 \mathrm{~N} \mathrm{KOH}$ standard $\mathrm{MeOH}$ solution was added until the color changed to pink. The volume of $\mathrm{KOH}$ standard solution $(2.1 \mathrm{~mL})$ was recorded and [COOH] in ssPES-OH was calculated. A similar procedure was repeated for ssPES-4. From the molecular weight of ssPES-OH, the fraction of ssPES terminated with $\mathrm{OH}$ groups at both chain ends 
and ssPES terminated with $\mathrm{OH}$ and $\mathrm{COOH}$ groups was calculated.

Reductive degradation of ssPES under reducing conditions was examined using GPC. ssPES-OH $(60 \mathrm{mg})$ was dissolved in THF $(5 \mathrm{~mL})$ under magnetic stirring. After an addition of different amounts of $\mathrm{Bu}_{3} \mathrm{P}$, aliquots of polymer solutions were taken at given time intervals to analyze molecular weight and molecular weight distribution of degraded products of ssPES-OH.

CMC of ssABPs in water was determined as follows: an aliquot of the purified, dried ssABP $(20 \mathrm{mg})$ was dissolved in THF (2.5 mL). The clear polymer solution was drop-wise added into deionized water $(20 \mathrm{~mL})$. The resulting dispersion was kept under stirring overnight to remove THF, allowing for colloidally stable micellar dispersion at a concentration of $1.0 \mathrm{mg} \mathrm{mL}^{-1}$. Then, aliquots of the aqueous stock solution were diluted with different amounts of deionized water, forming a series of ssABP solutions at different concentrations from $10^{-5}$ to $1.0 \mathrm{mg} \mathrm{mL}^{-1}$. A tensiometer was used to measure the pressure $\left(\mathrm{mN} \mathrm{m}^{-1}\right)$ of the solutions as follows: an aliquot of each ssABP solution $(600 \mu \mathrm{L})$ was carefully placed on each well and equilibrated before measurements. The tensiometer was calibrated using air and water.

For self-assembled micellization of ssABPs in water, a series of clear solutions of different amounts of purified, dried ssABP-1 was dissolved in THF $(2.5 \mathrm{~mL})$ and drop-wise added into deionized water $(20 \mathrm{~mL})$. The resulting dispersions were kept under stirring overnight to remove THF, resulting in colloidally stable micellar dispersions at different concentrations of ssABP1. Using the similar procedure, $10.5 \mathrm{mg} \mathrm{mL}^{-1}$ of micellar dispersion of ssABP-2 was prepared.

For release of Nile Red (NR) from thiol-responsive degradable micelles in water, NR-loaded micelles were prepared as follows: a stock solution of NR in THF $\left(5 \mathrm{mg} \mathrm{mL}^{-1}, 2 \mathrm{~mL}\right)$ and ssABP $(100 \mathrm{mg})$ was mixed with water $(10 \mathrm{~mL})$ under magnetic stirring for $30 \mathrm{~min}$. Non-dissolved NR was precipitated by centrifugation $\left(8000 \mathrm{rpm} \times 15 \mathrm{~min} \times 4{ }^{\circ} \mathrm{C}\right)$. The supernatant of NR-loaded micelles was divided into two aliquots of equivalent volume in fluorescence cuvettes. To an aliquot was added DTT (17 mg, $\mathrm{DTT} / \mathrm{SS}=1 / 1)$. Another aliquot was used as control without DTT. Their fluorescence spectra $\left(\lambda_{\mathrm{ex}}=480 \mathrm{~nm}\right)$ were measured at different time intervals and the fluorescence intensity at $\lambda_{\mathrm{em}}=$ $641 \mathrm{~nm}$ was monitored to construct the release profile of NR over degradation time. For GPC measurements of degraded products, water was removed from the mixtures by rotary evaporation. After polymeric residues were further dried in a vacuum oven at $50{ }^{\circ} \mathrm{C}$ overnight, they were then dissolved in THF to be analyzed by the GPC.

For cell viability using MTS assay, HEK293T cells were plated at $2 \times 10^{5}$ cells per well into a 96-well plate and incubated for $24 \mathrm{~h}$ in Dulbecco's Modified Eagle Medium (DMEM, $100 \mu \mathrm{L}$ ) containing $10 \%$ fetal bovine serum (FBS). They were then treated with various concentrations of ssABP-1 for another $48 \mathrm{~h}$. The cell viability was measured using CellTiter 96 Aqueous NonRadioactive Cell Proliferation Assay kit (Promega) according to the manufacturer's instruction. Briefly, a mixture of MTS and PMS solutions $(20 \mu \mathrm{L})$ was added into each well and the absorbance was recorded at $\lambda=490 \mathrm{~nm}$ after $4 \mathrm{~h}$ using FlexStation $\mathrm{II}^{384}$ (Molecular Devices).

\section{Results and discussion}

The new method employing a combination of polycondensation with ATRP allows for the synthesis of well-defined disulfidelabeled degradable ssABPs. The method includes three synthetic steps: (1) polycondensation through carbodiimide coupling or high temperature processes for synthesis of ssPES-OH, (2) bromination of ssPES-OH to ssPES-Br, and (3) ATRP for chain extension of ssPES-Br with water-soluble polymethacrylate. Degradation of ssPES-OH in the presence of reducing agent, thermal analysis, and micellization of ssABPs were systematically investigated. Release behavior and cell viability were also examined.

\section{Synthesis of ssPES-OH}

Two polycondensation processes to synthesize a series of reductively degradable ssPES-OH having disulfide linkages positioned repeatedly on the main chains were examined. Table 1 summarizes the experimental conditions, molecular weight data, and yield of final ssPES-OH homopolymers.

The utilization of carbodiimide coupling for the synthesis of multiblock copolymers has been reported ${ }^{47}$ In our experiments, two different diols including SS-DOH or C12-DOH reacted with SS-Dacid in the presence of DCC. White solids formed during reactions were removed by vacuum filtration and solvents were evaporated by rotary evaporation. For ssPES-OH containing $\mathrm{SS}-\mathrm{DOH}$, the precipitation of crude product from different solvents including hexane, $\mathrm{Et}_{2} \mathrm{O}$, and $\mathrm{MeOH}$ was examined. As presented in Table 1, molecular weights of precipitated ssPES-1, 2 , and 3 were $\sim 2000 \mathrm{~g} \mathrm{~mol}^{-1}$, while their yields were varied at $65-$ $75 \%$ with different solvents. ssPES-2 precipitated from $\mathrm{MeOH}$ had a lower yield and thus $\mathrm{MeOH}$ was selected for purification of ssPES-OH. Fig. 1 shows the ${ }^{1} \mathrm{H}-\mathrm{NMR}$ spectrum of ssPES-2, compared with those of starting materials of SS-DOH and SSDacid. A NMR signal at $3.9 \mathrm{ppm}$ corresponds to methylene protons (a) adjacent to terminal $\mathrm{OH}$ groups. The typical signal appeared at $4.4 \mathrm{ppm}$ that corresponds to methylene protons $\left(\mathrm{a}^{\prime}\right)$ adjacent to newly formed ester linkages.

For ssPES-OH containing C12-DOH, its crude product was precipitated from $\mathrm{MeOH}$, resulting in ssPES-4 with $M_{\mathrm{n}}=3500 \mathrm{~g}$ $\mathrm{mol}^{-1}$ at yield $=53 \%$. The structure of ssPES-4 was also confirmed by ${ }^{1} \mathrm{H}-\mathrm{NMR}$ (Fig. S1 $\dagger$ ). Its molecular weight was larger than that of ssPES containing SS-DOH, which is presumably attributed to longer chain length of $\mathrm{C} 12-\mathrm{DOH}$ than SS-DOH.

In an attempt to synthesize relatively high molecular weight ssPES, the high temperature polycondensation in the presence of $p$-TSA ${ }^{48}$ as a Lewis acid catalyst was examined. ${ }^{47}$ The $1: 1$ mole ratio of C12-DOH and SS-Dacid was mixed with $p$-TSA in bulk at $185{ }^{\circ} \mathrm{C}$. The amount of $p$-TSA defined as the ratio of $[\mathrm{C} 12-\mathrm{DOH}]_{0} /[p-\mathrm{TSA}]_{0}$ was varied with $50 / 1$ and $25 / 1$. Table 1 summarizes the results. For both polycondensation reactions with 50/1 (ssPES-5) and 25/1 ratio (ssPES-6), their molecular weights were $\sim 10000 \mathrm{~g} \mathrm{~mol}^{-1}$. When the polymerization time increased from 5 to $20 \mathrm{~h}$ with $[\mathrm{C} 12-\mathrm{DOH}]_{0} /[p-\mathrm{TSA}]_{0}=25 / 1$, the molecular weight of ssPES-7 increased to $19600 \mathrm{~g} \mathrm{~mol}^{-1}$, which is twice larger than that $\left(10000 \mathrm{~g} \mathrm{~mol}^{-1}\right)$ of ssPES-6 $(5 \mathrm{~h})$. These results indicate that the high temperature process enabled the 
Table 1 Polycondensation for synthesis of reductively degradable ssPES-OH homopolymers

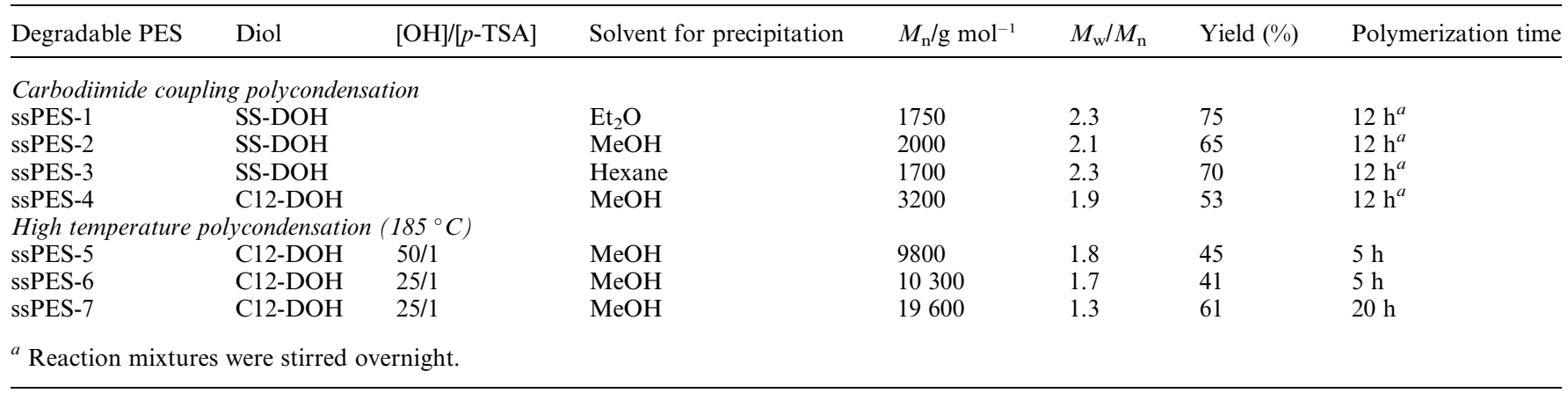

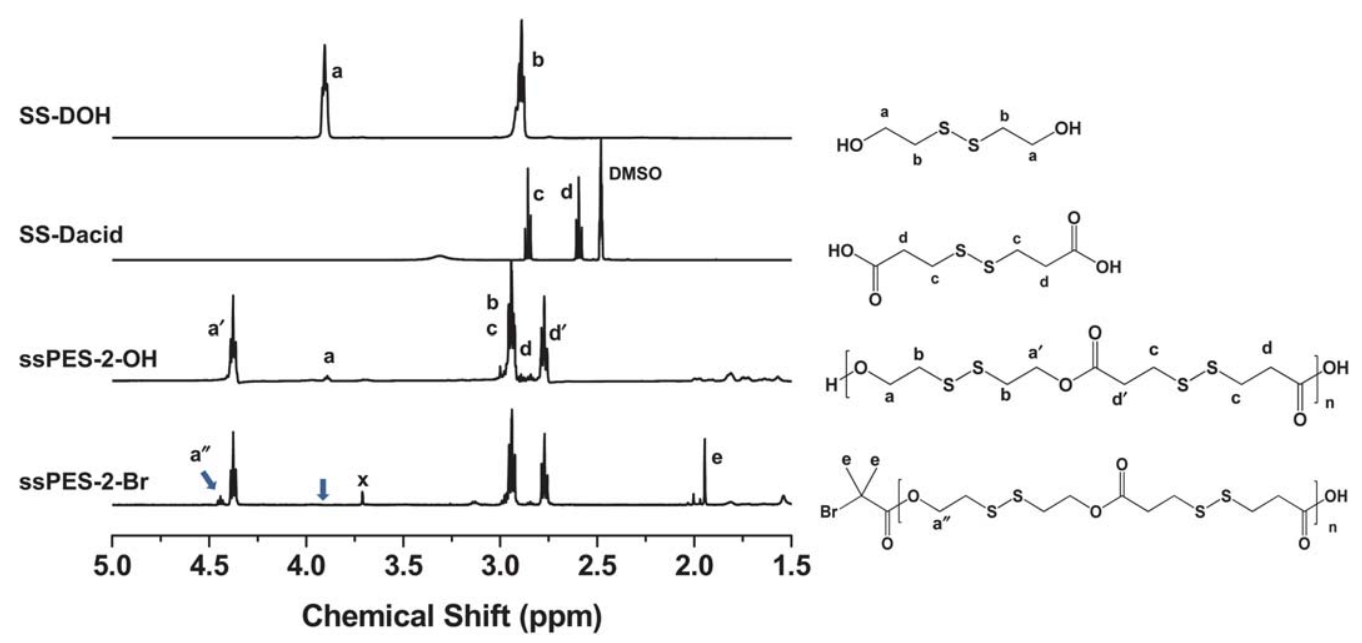

Fig. $1{ }^{1} \mathrm{H}-\mathrm{NMR}$ spectra of SS-DOH, ssPES-2, and ssPES-2-Br in $\mathrm{CDCl}_{3}$ and SS-Dacid in DMSO-d .

Table 2 Acid-base titration results of ssPES-OH homopolymers

\begin{tabular}{llllll}
\hline ssPES & Diol & Sample $/ \mathrm{mg}$ & $\mathrm{Vol}^{a} / \mathrm{mL}$ & {$[\mathrm{COOH}]_{\mathrm{o}} / \mathrm{mmol}$} & {$[\mathrm{COOH}]_{\mathrm{end}} / \mathrm{mmol}$} \\
\hline ssPES-1 & SS-DOH & 503.6 & 2.1 & 2.74 & 0.22 \\
ssPES-4 & C12-DOH & 445.8 & 2.1 & 2.08 & 0.22
\end{tabular}

${ }^{a}$ Volume of $0.102 \mathrm{~N} \mathrm{KOH}$ standard solution in $\mathrm{MeOH}$ at change in color. ${ }^{b}$ Extent of reaction $=[\mathrm{COOH}]_{\text {reacted }} /[\mathrm{COOH}]_{0}$.

synthesis of relatively high molecular weight ssPES-OH. In addition, polymerization time is an important parameter that significantly increases the molecular weight of ssPES. However, when SS-DOH was mixed with SS-Dacid at the temperature of $185^{\circ} \mathrm{C}$, no significant amounts of ssPES were obtained.

Table 2 presents the availability of terminal hydroxyl $(-\mathrm{OH})$ group of ssPES-OH determined by acid-base titration in a mixture of $1 / 2 \mathrm{v} / \mathrm{v} \mathrm{MeOH} / \mathrm{THF}$. The titration results, with the $M_{\mathrm{n}}$ values determined by GPC (Table 1), allowed for estimating the fraction of ssPES having terminal $\mathrm{OH}$ and $\mathrm{COOH}$ groups. It was $84 \%$ for ssPES-3 and $76 \%$ for ssPES-4.

\section{Reductive degradation of ssPES-OH in reducing environments}

The reductive cleavage of disulfide linkages of ssPES-OH homopolymers was investigated with $\mathrm{Bu}_{3} \mathrm{P}$, an organic reducing agent. Aliquots of ssPES-OH dissolved in THF were magnetically stirred with different amounts of $\mathrm{Bu}_{3} \mathrm{P}$ defined as the mole ratio of $\mathrm{Bu}_{3} \mathrm{P} /$ disulfide ( $\mathrm{SS}$ ). GPC was used to measure the molecular weight of ssPES-OH with degradation time.

Fig. S $2 \uparrow$ shows overlaid GPC traces of ssPES- 2 and ssPES-4 in the absence and presence of different amounts of $\mathrm{Bu}_{3} \mathrm{P}$. In the presence of $\mathrm{Bu}_{3} \mathrm{P}, \mathrm{GPC}$ traces evolved to low molecular weight region over time, suggesting the decrease in molecular weight of ssPES-OH due to the cleavage of SS linkages upon degradation

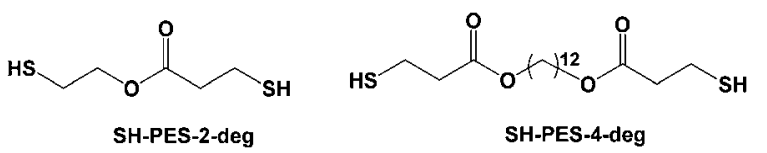

Scheme 2 Chemical structures of corresponding thiols degraded from ssPES-2 and ssPES-4 in the presence of $\mathrm{Bu}_{3} \mathrm{P}$. 
of ssPES-OH. It can be assumed that when SS linkages are completely cleaved to their corresponding thiols, the degraded product could be SH-PES-2-deg with $M_{\mathrm{n}}=182 \mathrm{~g} \mathrm{~mol}^{-1}$ for ssPES-2 containing SS-DOH and SH-PES-4-deg with $M_{\mathrm{n}}=$ $412 \mathrm{~g} \mathrm{~mol}^{-1}$ for ssPES-4 containing C12-DOH (Scheme 2). With the molecular weights of the degraded thiols, the molecular weights of degraded ssPES at different times were normalized, resulting in " $\%$ Remaining ssPES" as the extent of degradation of ssPES.

Fig. 2a shows the $\%$ Remaining ssPES-2 (containing SS-DOH) in the absence and presence of different amounts of $\mathrm{Bu}_{3} \mathrm{P}$ (different ratios of $\mathrm{Bu}_{3} \mathrm{P} / \mathrm{SS}$ ) over time. In the absence of $\mathrm{Bu}_{3} \mathrm{P}$, the $\%$ Remaining ssPES-2 did not change, suggesting no significant degradation of ssPES-2. When $\mathrm{Bu}_{3} \mathrm{P}$ was added, $\%$ Remaining ssPES-2 decreased with degradation time. With an increasing amount of $\mathrm{Bu}_{3} \mathrm{P}$, the decrease in $\%$ Remaining ssPES-2 over time was enhanced. Similar results were observed for ssPES4 containing $\mathrm{C} 12-\mathrm{DOH}$ with slower decrease in $\%$ Remaining ssPES-4 (Fig. 2b). Fig. 2c compares degradation behavior of ssPES-2 with ssPES-4 as a function of different ratios of $\mathrm{Bu}_{3} \mathrm{P} / \mathrm{SS}$ at given degradation times. First of all, faster degradation was observed with an increasing amount of $\mathrm{Bu}_{3} \mathrm{P}$. For ssPES-2, \% Remaining ssPES decreased from $100 \%$ for $0 / 1$ ratio $\left(\right.$ no $\left.\mathrm{Bu}_{3} \mathrm{P}\right)$ to $60 \%$ for $0.2 / 1,40 \%$ for $0.5 / 1$, and further to $<10 \%$ for $1 / 1$ after 30 min. For ssPES-4 containing C12-DOH, \%Remaining ssPES decreased to $60 \%$ for $1 / 1$ and $40 \%$ for $2 / 1$ after $2 \mathrm{~h}$ and $30 \%$ for $1 / 1$ and $20 \%$ for $2 / 1$ after $6 \mathrm{~h}$. Secondly, the degradation of ssPES-2 containing SS-DOH was much faster than that of ssPES-4 containing C12-DOH. For example, with $\mathrm{Bu}_{3} \mathrm{P} / \mathrm{SS}=$ $1 / 1$, the $\%$ Remaining ssPES was $<10 \%$ for ssPES- 2 after $30 \mathrm{~min}$, while it was $60 \%$ after $2 \mathrm{~h}$ and $30 \%$ after $6 \mathrm{~h}$ for ssPES- 4 . The faster degradation of ssPES-2 could be attributed to more SS linkages in ssPES-2 chains from SS-DOH.

Due to fast degradation of ssPES containing SS-DOH, further experiments for chain extension, thermal analysis, micellization, release, and cell viability were performed with only ssPES containing SS-DOH, particularly ssPES-2.

\section{Synthesis of ssABP using ATRP}

ATRP was utilized for the chain extension of ssPES-2 with water-soluble polymethacrylate, allowing for the synthesis of well-defined degradable ssABPs. First, bromination of ssPES$\mathrm{OH}$ was completed by its reaction with $\mathrm{Br}-\mathrm{iBuBr}$ in the presence of $\mathrm{Et}_{3} \mathrm{~N}$, yielding ssPES-2-Br as an ATRP macroinitiator. The crude product was washed with both aqueous acidic and alkali solutions to remove water-soluble species as well as to hydrolyze anhydrides possibly formed by the reaction of terminal carboxylic acid with $\mathrm{Br}-\mathrm{iBuBr}$. After precipitation from $\mathrm{MeOH}$, the molecular weight of ssPES-Br increased to $M_{\mathrm{n}}=2400 \mathrm{~g} \mathrm{~mol}^{-1}$
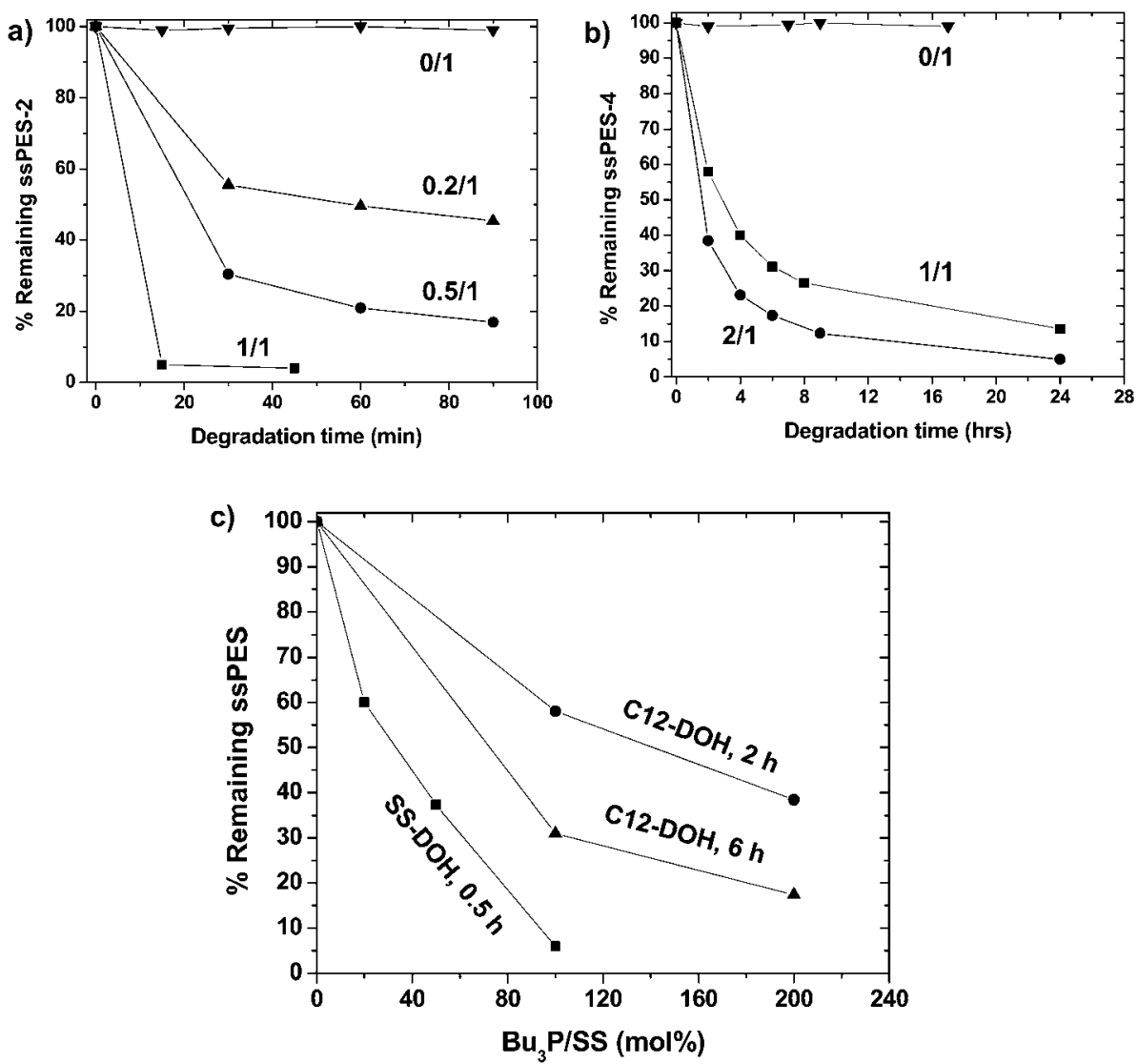

Fig. 2 Degradation of ssPES-2 containing SS-DOH (a) and ssPES-4 containing C12-DOH (b) with different amounts of $\mathrm{Bu}_{3} \mathrm{P}$ defined as the mole ratio of $\mathrm{Bu}_{3} \mathrm{P} / \mathrm{SS}$ over degradation time (a and $\mathrm{b}$ ), and over $\mathrm{Bu}_{3} \mathrm{P} / \mathrm{SS}(\mathrm{c})$. 
with $M_{\mathrm{w}} / M_{\mathrm{n}}=1.5$. Such increase is probably due to the removal of low molecular weight species. ${ }^{1} \mathrm{H}-\mathrm{NMR}$ confirmed successful bromination. As seen in Fig. 1, the signal at $3.9 \mathrm{ppm}$ (a) for methylene protons adjacent to terminal $\mathrm{OH}$ group of ssPES-2 disappeared; a new peak at $4.5 \mathrm{ppm}\left(\mathrm{a}^{\prime \prime}\right)$ corresponding to methylene protons to bromoester group appeared.

Next, a series of ATRP of a water-soluble oligo(ethylene glycol) monomethyl ether methacrylate (OEOMA) in the presence of ssPES-2-Br in anisole at $47^{\circ} \mathrm{C}$ was examined. Ligands for $\mathrm{CuBr}$ such as HMTETA and bpy and a ratio of [OEOMA $]_{0} /$ $[\text { ssPES-2-Br }]_{0}=50 / 1$ and 100/1 were varied. As summarized in Table 3, all three polymerizations were relatively fast, reaching $>80 \%$ conversion within 2-3 h. However, ATRP-1 with 50/1 and HMTETA was lightly faster than ATRP-2 with 50/1 and bpy, but slower than ATRP-3 with 100/1 and HMTETA. As shown in Fig. 3, the three polymerizations were first order, indicating a constant concentration of active centers during the polymerizations. The molecular weight increased with conversion and the polydispersity remained low, $M_{\mathrm{w}} / M_{\mathrm{n}}<1.4$ up to $60 \%$ monomer conversion.

In another set of experiment, the effect of the presence of a small amount of $\mathrm{CuBr}_{2}$ deactivator on the course of the ATRP of OEOMA was examined. A $10 \% \mathrm{CuBr}_{2}$ to $\mathrm{CuBr}$ activator was introduced to slow down polymerization. As seen in Fig. 4a, the polymerization is first order with an induction period of $1 \mathrm{~h}$. The molecular weight increased with conversion and its values were somewhat smaller than theoretically predicted (Fig. 4b). The polydispersity remained low, $M_{\mathrm{w}} / M_{\mathrm{n}}<1.4$ up to $60 \%$ monomer conversion. GPC traces show bimodal distributions (Fig. 4c). The high molecular weight species had peak molecular weights twice higher than those of the lower molecular weight species. The higher molecular weight species could be formed from difunctional macroinitiators (Br-ssABP-Br).

Well-defined ssABPs with different chain lengths of watersoluble POEOMA block were purified as described in the Experimental section. Table 4 summarizes their properties including molecular weight, $\mathrm{CMC}$, and particle size data as well as the amount of ssPES-2 in block copolymers. Thermal analysis, micellization, release behavior, and cell viability of these block copolymers were examined.

\section{Thermal analysis of ssPES and ssABP}

Thermal properties of ssPES and block copolymers (ssABPs) were characterized by utilizing DSC. As seen in Fig. 5a, the DSC diagram of ssPES-2 shows a strong endothermic melting peak at $T_{\mathrm{m}}=\sim 34{ }^{\circ} \mathrm{C}$. ssABPs consist of hydrophobic ssPES block and hydrophilic POEOMA block, both of which are immiscible. Fig. 5b shows the DSC diagrams of ssABPs with different molecular weights of POEOMA block. For ssABP-1 with $M_{\mathrm{n}}=$ $11000 \mathrm{~g} \mathrm{~mol}^{-1}$ containing $17 \%$ ssPES, the diagram shows the
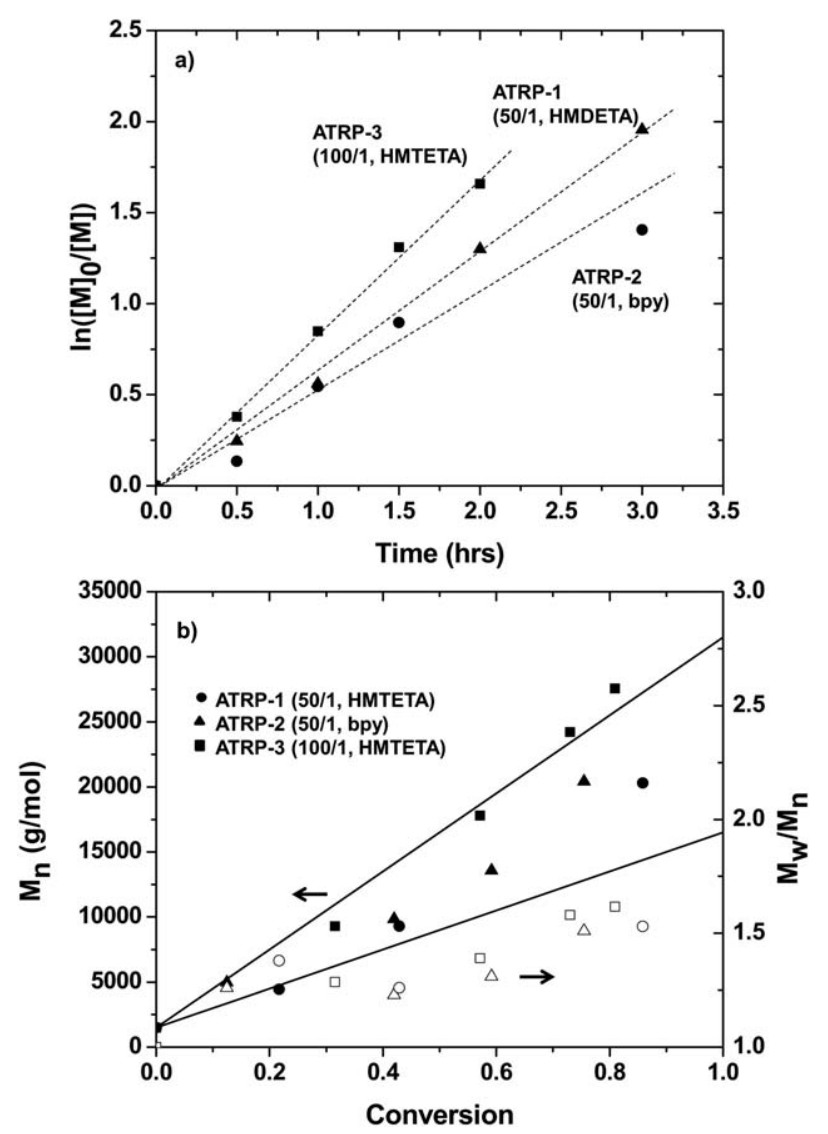

Fig. 3 First-order kinetic plot (a) and evolution of molecular weight and molecular weight distribution with conversion (b) for ATRP of OEOMA in the presence of ssPES-2-Br in anisole at $47^{\circ} \mathrm{C}$. Conditions: [OEOMA $]_{0} /$ $[\text { ssPES-2-Br }]_{0} /[\text { ligand }]_{0}=x / 1 / y$ where $x=50$ and 100 and $y=0.5$ for HMTEMA and 1 for bpy and OEOMA/anisole $=54 \mathrm{wt} \%$. The dotted lines in (a) are linear fits, and the straight lines in (b) are the theoretically predicted molecular weight over conversion.

melting peak at $T_{\mathrm{m}}=\sim 34^{\circ} \mathrm{C}$ contributed from crystalline ssPES block and a glass transition at $T_{\mathrm{g}}=\sim-33{ }^{\circ} \mathrm{C}$ from amorphous POEOMA block. For ssABP-2 with larger POEOMA block $\left(M_{\mathrm{n}}=17000 \mathrm{~g} \mathrm{~mol}^{-1}\right.$ and $11 \%$ ssPES), both $T_{\mathrm{m}}$ and $T_{\mathrm{g}}$ were observed. However, weak endothermic $T_{\mathrm{m}}$ peak appeared due to the less amount of crystalline ssPES in the block copolymer.

\section{Aqueous micellization of ssABP}

ssABP block copolymers are amphiphilic and can form micelles consisting of a hydrophobic ssPES core surrounded with relatively long hydrophilic POEOMA coronas. CMC values of ssABPs were determined by tensiometry. A stock solution of each ssABP in water at the concentration of $1.0 \mathrm{mg} \mathrm{mL}^{-1}$ was

Table 3 A series of ATRP of OEOMA in the presence of ssPES-2-Br macroinitiator

\begin{tabular}{llllll}
\hline ssABP & {$[\mathrm{OEOMA}]_{0} /[\mathrm{ssPES}-2-\mathrm{Br}]_{0}$} & Ligand & Time/h & Conversion/\% & $M_{\mathrm{n}} / \mathrm{g} \mathrm{mol}{ }^{-1}$ \\
\hline ATRP-1 & $50 / 1$ & HMTETA & 3 & 86 & 20300 \\
ATRP-2 & $50 / 1$ & bpy & 3 & 82 & 22600 \\
ATRP-3 & $100 / 1$ & HMTETA & 2 & 81 & 1.53 \\
ATRP-4 & $50 / 1$ & PMDETA & 3 & 85 & 27500 \\
\hline
\end{tabular}



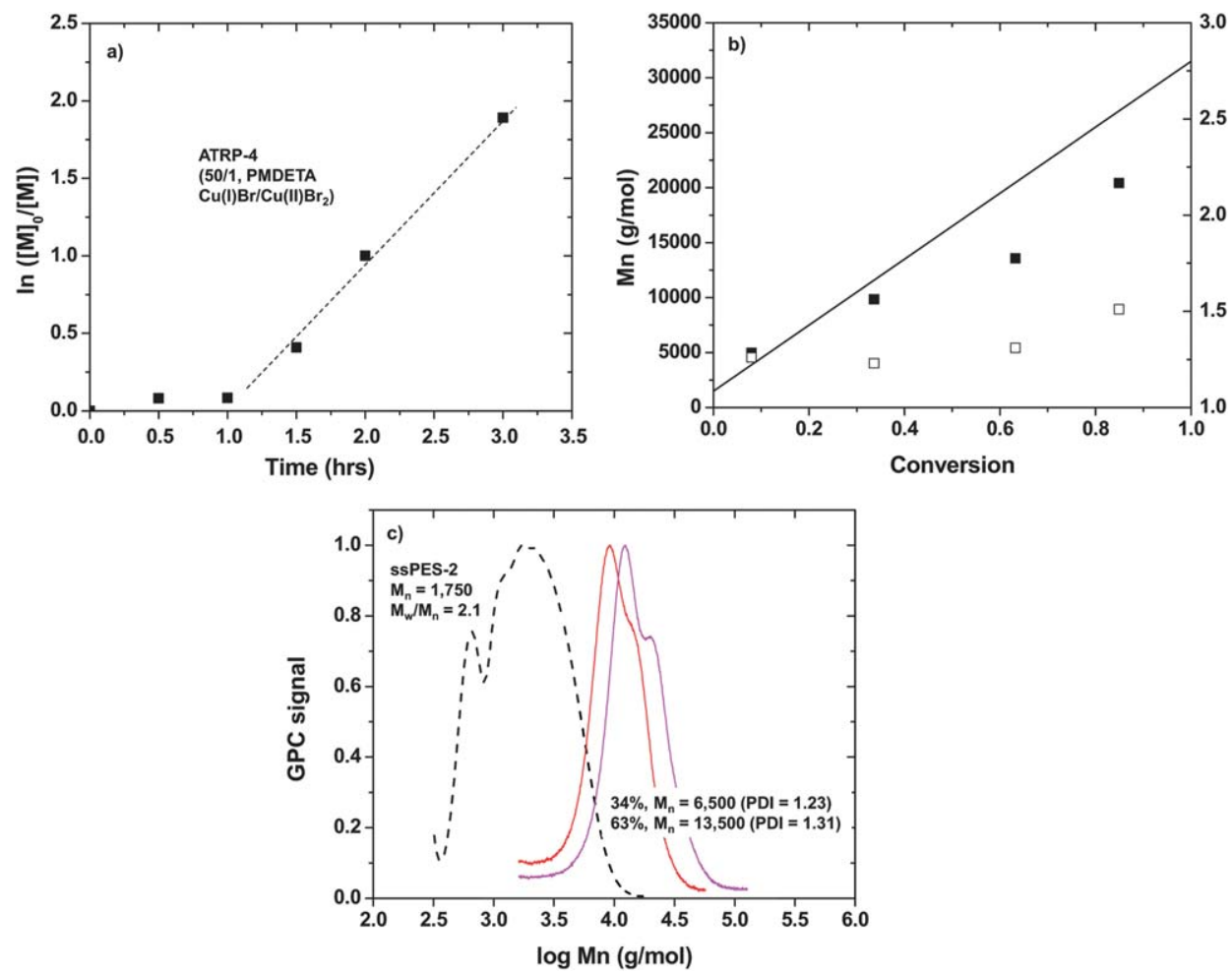

Fig. 4 First-order kinetic plot (a), evolution of molecular weight and molecular weight distribution (b), and GPC traces (c) over conversion for ATRP of OEOMA in the presence of ssPES-2-Br in anisole at $47^{\circ} \mathrm{C}$. Conditions: $[\mathrm{OEOMA}]_{0} /[\mathrm{ssPES}-2-\mathrm{Br}]_{0} /[\mathrm{CuBr} / \mathrm{PMDETA}]_{0} /\left[\mathrm{CuBr}{ }_{2} / \mathrm{PMDETA}\right]_{0}=50 / 1 / 0.5 /$ 0.05 and OEOMA/anisole $=54 \mathrm{wt} \%$. The dotted line in (a) is linear fit, and the straight line in (b) is the theoretically predicted molecular weight over conversion.

Table 4 Properties of well-defined ssABPs and their micelles

\begin{tabular}{|c|c|c|c|c|c|c|}
\hline & $M_{\mathrm{n}} / \mathrm{g} \mathrm{mol}^{-1}$ & $M_{\mathrm{w}} / M_{\mathrm{n}}$ & ssPES (\%) & $\mathrm{CMC}^{a} / \mu \mathrm{g} \mathrm{mL}^{-1}$ & \multicolumn{2}{|l|}{ Diameter $/ \mathrm{nm}^{b}$} \\
\hline ssPES-2 & 2000 & 2.10 & 100 & Precipitated & & \\
\hline ssABP-1 & 11000 & 1.27 & 16.6 & 12 & 22.1 & 138.0 \\
\hline ssABP-2 & 17000 & 1.47 & 10.6 & 8 & 12.2 & 61.1 \\
\hline
\end{tabular}

prepared by mixing ssABP in THF with water, followed by evaporation of THF. Aliquots of each stock solution were diluted with various amounts of water. Fig. 6 a shows the plots of surface pressure values measured using a tensiometer $v s$. various concentrations of ssABPs in water. For ssABP-1, pressure does not change at its lower concentration; however it increased with an increasing concentration of ssABP-1, and then slowly increased with further increase in the amount of ssABP-1. From two equations obtained by fitting each dataset to linear relationships, the CMC of ssABP-1 with $M_{\mathrm{n}}=11000 \mathrm{~g} \mathrm{~mol}^{-1}$ was

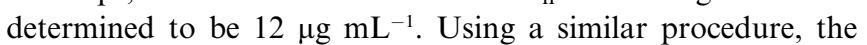
CMC of ssABP-2 with $M_{\mathrm{n}}=17000 \mathrm{~g} \mathrm{~mol}^{-1}$ was $8 \mu \mathrm{g} \mathrm{mL}^{-1}$. These results indicate that the CMC decreased as the corona chain length increased.

The particle size and size distribution of micelles of ssABPs were determined using dynamic light scattering (DLS) at the concentrations above the CMC. The solvent evaporation method was utilized for micellization. ${ }^{49,50}$ As expected, the hydrophobic ssPES-2 homopolymer itself did not form micelles, but aggregated (Table 4). ssPES- $b$-POEOMA block copolymers formed stable micelles. First, the effect of corona size (chain length of POEOMA block) on particle size and size distribution was examined at a $10 \mathrm{mg} \mathrm{mL}^{-1}$ micellar concentration. Fig. $6 \mathrm{~b}$ shows CONTIN plots for micelles of ssABP-1 with $M_{\mathrm{n}}=$ $11000 \mathrm{~g} \mathrm{~mol}^{-1}$ and ssABP-2 with $M_{\mathrm{n}}=17000 \mathrm{~g} \mathrm{~mol}^{-1}$. The micelles contained two populations with small and large particle sizes, as reported for micelles of poly(lactide- $c o$-glycolic acid)- $b$ POEMA in water. ${ }^{50}$ As compared in Table 4 , the average particle size of large population was calculated to be $138.0 \mathrm{~nm}$ for ssABP1 and $61.1 \mathrm{~nm}$ for ssABP-2. The small particles with diameter $=$ 12-22 $\mathrm{nm}$ are probably attributed to the presence of unimers. Note that the concentration of $10 \mathrm{mg} \mathrm{mL}^{-1}$ is significantly higher than the CMC $\left(0.012 \mathrm{mg} \mathrm{mL}^{-1}\right)$. When a micellar dispersion of ssABP-1 at the concentration of $1.0 \mathrm{mg} \mathrm{mL}^{-1}$ was diluted by 

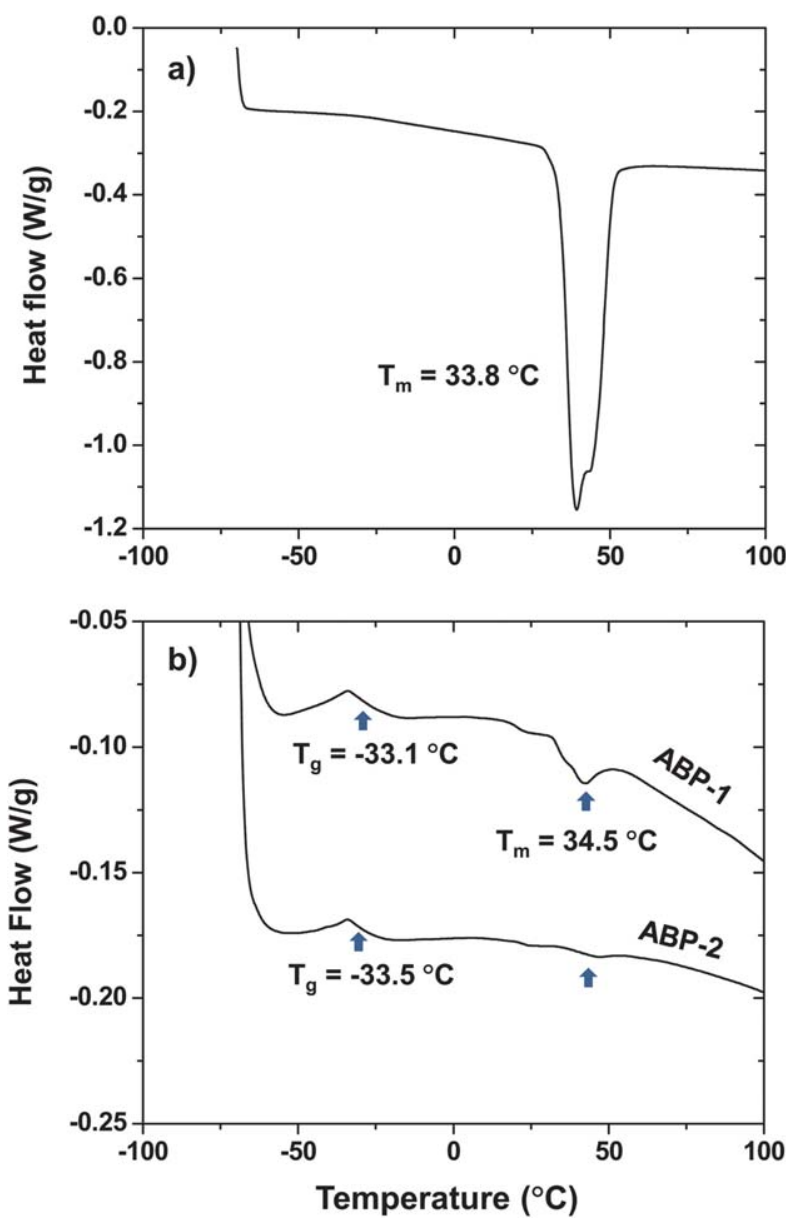

Fig. 5 DSC diagrams of ssPES homopolymer (ssPES-2) (a) and ssABP-1 and ssABP-2 (b).

100 folds (eventually, reaching $0.01 \mathrm{mg} \mathrm{mL}^{-1}$, close to the $\mathrm{CMC}$ ), the DLS indicates one population with an average diameter of $71.2 \mathrm{~nm}$ (Fig. S4†).

Next, the effect of the amount of ssABP-1 (having $M_{\mathrm{n}}=$ $11000 \mathrm{~g} \mathrm{~mol}^{-1}$ ) on the formation of micelles in water was examined. A series of micellar dispersions with different amounts

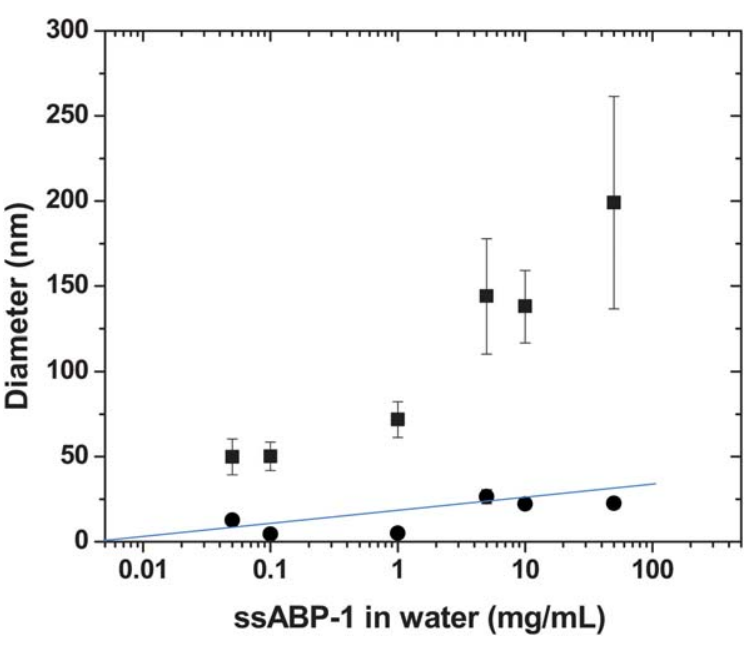

Fig. 7 Adjustment of the diameter of micelles with varying concentrations of ssABP-1 in water. The line was added for better visualization on an increase in the size of small particles.

of ssABP-1 ranging from 0.05 to $50 \mathrm{mg} \mathrm{mL}^{-1}$ in water were prepared. Note that the amount of THF to dissolve the different amounts of ssABP-1 was kept constant. Their CONTIN plots based on \%intensity in water also show two populations (Fig. S3 $)$ ). As seen in Fig. 7, the diameters of both populations increased with an increasing amount of ssABP-1 in water: 50 to $200 \mathrm{~nm}$ for large population and 5 to $22 \mathrm{~nm}$ for small population. Such increase in particle sizes could be attributed to higher concentration of ssABP-1 in THF solution, thus leading to higher viscosity and slower diffusion of the organic phase into aqueous phase. ${ }^{49,51}$ These results suggest that the particle size of ssABP-based micelles could be adjusted by varying the size of POEOMA corona and concentration of ssABP in water.

\section{Release of encapsulated model drug upon degradation of micelles}

The release of a fluorescent dye as a model encapsulated drug upon degradation of ssABP-based micelles in response to DTT, a water-soluble thiol, in aqueous solutions was examined. Nile Red (NR) is hydrophobic and has been utilized as a hydrophobic
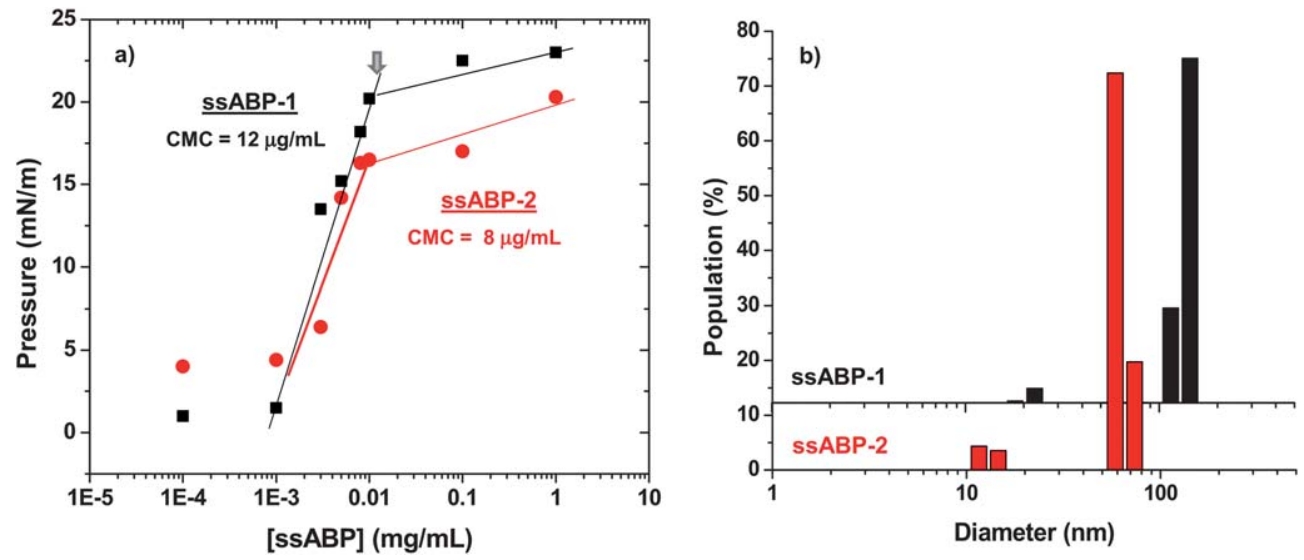

Fig. 6 Determination of CMC by tensiometry (a) and CONTIN plots based on \%intensity for micelles (b) of ssABP-1 with $M_{\mathrm{n}}=11000 \mathrm{~g}$ mol ${ }^{-1}$ and ssABP-2 with $M_{\mathrm{n}}=17000 \mathrm{~g} \mathrm{~mol}^{-1}$ in water. 

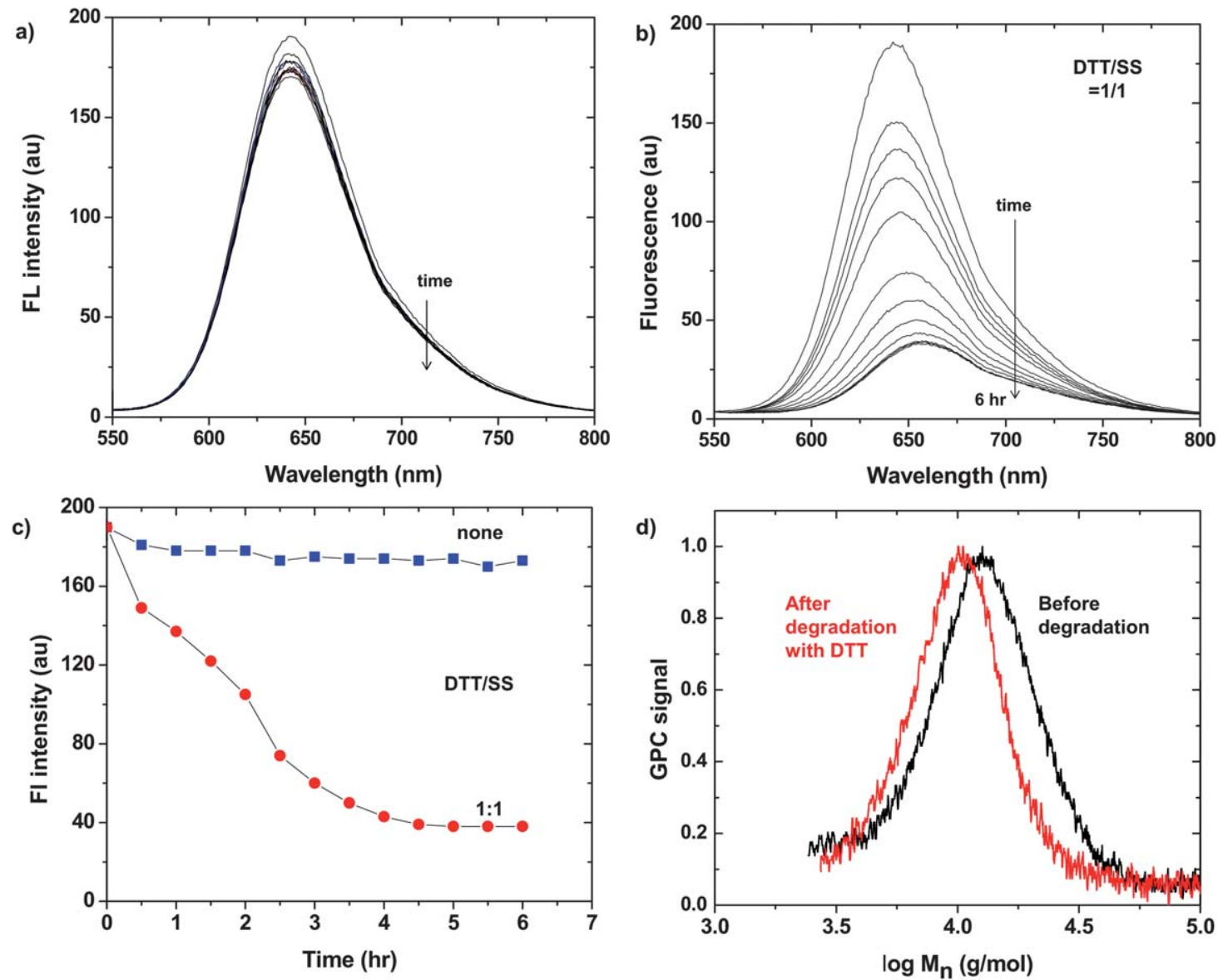

Fig. 8 For ssABP-1 micelles, overlaid fluorescence spectra $\left(\lambda_{\mathrm{ex}}=480 \mathrm{~nm}\right)$ of NR in a mixture of water/THF $(82 / 18 \mathrm{w} / \mathrm{w})$ without (a) and with DTT whose amount is defined as the mole ratio of DTT/SS $=1 / 1$ (b), release profile of NR from NR-loaded micelles over degradation time (c), and GPC traces before and after degradation (d).

model encapsulate. ${ }^{52,53}$ ssABP-1 and NR were dissolved in a mixture of water/THF $(82 / 18 \mathrm{w} / \mathrm{w})$. After the removal of nondissolved NR using centrifugation, the supernatant was divided into two equivalent aliquots in fluorescence cuvettes: one aliquot without DTT as a control and the other aliquot mixed with DTT whose amount is defined as the mole ratio of DTT/SS $=1 / 1$. Their fluorescence spectra were measured at given time intervals to monitor the fluorescence intensity at $\lambda_{\mathrm{em}}=641 \mathrm{~nm}$ (Fig. $8 \mathrm{a}$ and b). No significant weight loss possibly caused by the evaporation of THF during measurements was observed during experiments. Fig. 8c shows the release profile of NR from NR-loaded micelles over degradation time. In the absence of DTT, NR can be either confined in small micellar cores or dissolved in water/THF mixture. No significant change in the fluorescence intensity was observed over $6 \mathrm{~h}$, suggesting no release as well as no photobleaching of significant amount of NR. When DTT is added, NRloaded micelles degrade to the corresponding water-soluble HSPOEOMA, as confirmed by GPC traces in Fig. 8d. Upon micellar degradation, NR was released to the mixture of water/THF and its fluorescence intensity decreased over time, which is attributed to quenching by water molecules, not by photobleaching. ${ }^{53}$ The results suggest faster release of NR in the presence of DTT; $>85 \%$ NR was released within $3 \mathrm{~h}$. These results confirm that thiolresponsive degradation enabled fast and controlled release of encapsulated molecules.

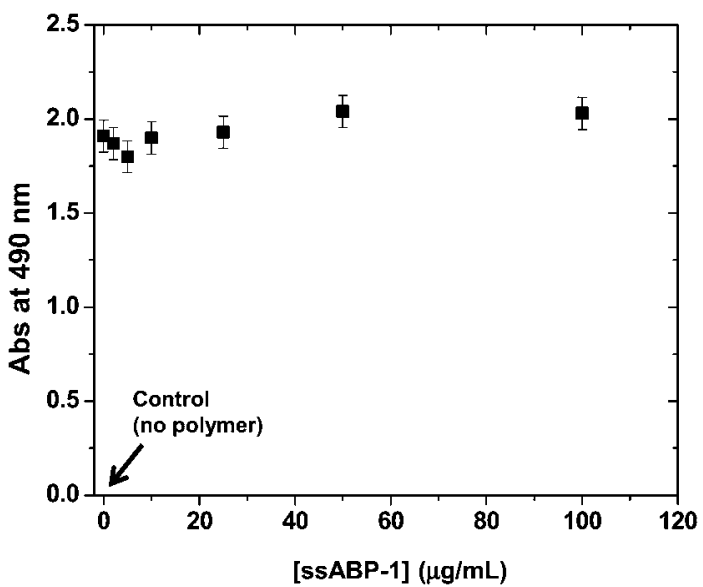

Fig. 9 Viability of HEK cells cultured with various amounts of ssABP-1 for $48 \mathrm{~h}$

\section{Cell viability}

The MTS assay (a calorimetric method to measure cell viability) was used to examine the cytotoxicity of ssABPs. Aliquots of different concentrations of ssABP-1 were cultured with human embryonic kidney HEK293T cells. After $48 \mathrm{~h}$ incubation, the absorbance was measured using absorbance-based plate reader. 
As seen in Fig. 9, the absorbance values in the presence of different amounts of ssABP-1 up to $100 \mu \mathrm{g} \mathrm{mL}^{-1}$ were similar to that without ssABP-1 as a control. This result suggests that ssABP block copolymers are nontoxic to HEK293 cells.

\section{Conclusion}

Well-defined reductively degradable ssABPs were synthesized by the new method employing a combination of polycondensation and ATRP. These well-defined block copolymers consist of a reductively degradable ssPES block labeled repeatedly with disulfide linkages on the main chain at regular intervals, thus exhibiting fast degradation in reducing environments, and a water-soluble POEOMA block, ensuring water-solubility and biocompatibility.

Polycondensation through both carbodiimide coupling and high temperature processes allowed for the synthesis of disulfidelabeled ssPES-OH containing SS-DOH and C12-DOH; the high temperature process resulted in relative high molecular weight ssPES. The cleavage of disulfide linkages in reducing conditions led to the degradation of ssPES homopolymers. GPC results monitoring the decrease in molecular weight of their degraded products suggest that faster degradation was obtained with increasing amounts of disulfides in ssPES and reducing agents. ATRP of OEOMA in the presence of brominated ssPES-Br containing SS-DOH was controlled, allowing for the synthesis of ssPES- $b$-POEOMA copolymers (ssABPs). All polymerizations were first-order; molecular weight increased with conversion; and molecular weight distribution remained $<1.4$ up to $60 \%$ conversion.

Well-defined ssABPs were amphiphilic to form stable core/ shell micelles. Their CMC values were in range of $8-12 \mu \mathrm{gL}^{-1}$; such low CMC values could be advantageous for drug delivery applications in vivo, because such micelles can offer great colloidal stability upon dilution under physiological conditions. The particle size increased with an increasing amount of ssABP in water and hydrophilic POEOMA block length. The results of release behavior and cell viability suggest that thiol-responsive degradation of ssABP-micelles enabled fast release of encapsulated model drugs and the ssABP copolymers are nontoxic to cells and biocompatible.

These results are promising in that ssABP micelles hold potential for fast and controlled drug delivery. The ability to control micelle size could be advantageous for varying loading levels of therapeutics as well as cellular uptake and clearance. Moreover, tunable degradation of drug-loaded ssABP micelles in response to thiols should enable the controlled release of encapsulated therapeutics in targeted diseased cells.

\section{Acknowledgements}

Financial supports from Concordia University (Start-Up) and NSERC Canada (Discovery Grant) are greatly appreciated. JKO is entitled Tier II Canada Research Chair in Nanobioscience. Authors thank Prof. Cuccia in the Department of Chemistry and Biochemistry at Concordia University for his helpful discussion and kind donation of GPC as well as Mr Patrick Pinel for his initial work on polyester degradation.

\section{References}

1 A. S. Mikhail and C. Allen, J. Controlled Release, 2009, 138, 214.

2 A. Blanazs, S. P. Armes and A. J. Ryan, Macromol. Rapid Commun., 2009, 30, 267.

3 N. Nishiyama and K. Kataoka, Adv. Polym. Sci., 2006, 193, 67.

4 A. Harada and K. Kataoka, Prog. Polym. Sci., 2006, 31, 949.

5 J. K. Oh, Soft Matter, 2011, 7, 5096.

6 C. Alexander and K. M. Shakesheff, Adv. Mater., 2006, 18, 3321.

7 N. Rapoport, Prog. Polym. Sci., 2007, 32, 962.

8 M. Motornov, Y. Roiter, I. Tokarev and S. Minko, Prog. Polym. Sci., 2010, 35, 174.

9 J.-M. Schumers, C.-A. Fustin and J.-F. Gohy, Macromol. Rapid Commun., 2010, 31, 1588.

10 N. V. Tsarevsky and K. Matyjaszewski, Macromolecules, 2005, 38, 3087.

11 J. K. Oh, C. Tang, H. Gao, N. V. Tsarevsky and K. Matyjaszewski, J. Am. Chem. Soc., 2006, 128, 5578.

12 N. V. Tsarevsky and K. Matyjaszewski, Macromolecules, 2002, 35, 9009.

13 L. Zhang, W. Liu, L. Lin, D. Chen and M. H. Stenzel, Biomacromolecules, 2008, 9, 3321.

14 R. A. Petros, P. A. Ropp and J. M. DeSimone, J. Am. Chem. Soc., 2008, 130, 5008.

15 C. Li, J. Madsen, S. P. Armes and A. L. Lewis, Angew. Chem., Int. $E d ., 2006,45,3510$.

16 S. Carelli, A. Ceriotti, A. Cabibbo, G. Fassina, M. Ruvo and R. Sitia, Science, 1997, 277, 1681.

17 Y. Li, B. S. Lokitz, S. P. Armes and C. L. McCormick, Macromolecules, 2006, 39, 2726.

18 L. Zhang, J. Bernard, T. P. Davis, C. Barner-Kowollik and M. H. Stenzel, Macromol. Rapid Commun., 2008, 29, 123.

19 Y. Li, W. Du, G. Sun and K. L. Wooley, Macromolecules, 2008, 41, 6605.

20 C. F. van Nostrum, Soft Matter, 2011, 7, 3246.

21 M. Kang and B. Moon, Macromolecules, 2009, 42, 455.

22 J. S. Katz, S. Zhong, B. G. Ricart, D. J. Pochan, D. A. Hammer and J. A. Burdick, J. Am. Chem. Soc., 2010, 132, 3654.

23 J.-M. Schumers, J.-F. Gohy and C.-A. Fustin, Polym. Chem., 2010, 1, 161.

24 T.-B. Ren, Y. Feng, Z.-H. Zhang, L. Li and Y.-Y. Li, Soft Matter, 2011, 7, 2329.

25 R. P. Brinkhuis, T. R. Visser, F. P. J. T. Rutjes and J. C. M. van Hest, Polym. Chem., 2011, 2, 550.

26 Y. Wang, H. Xu and X. Zhang, Adv. Mater., 2009, 21, 2849.

27 X. Huang, F. Du, R. Ju and Z. Li, Macromol. Rapid Commun., 2007, 28, 597.

28 J. Babin, M. Pelletier, M. Lepage, J.-F. Allard, D. Morris and Y. Zhao, Angew. Chem., Int. Ed., 2009, 48, 3329.

29 X. Huang, F. Du, J. Cheng, Y. Dong, D. Liang, S. Ji, S.-S. Lin and Z. Li, Macromolecules, 2009, 42, 783.

30 W. Chen, F. Meng, F. Li, S.-J. Ji and Z. Zhong, Biomacromolecules, 2009, 10, 1727.

31 N. Fomina, C. McFearin, M. Sermsakdi, O. Edigin and A. Almutairi, J. Am. Chem. Soc., 2010, 132, 9540.

32 D. Han, X. Tong and Y. Zhao, Macromolecules, 2011, 44, 437.

33 K. A. Davis and K. Matyjaszewski, Adv. Polym. Sci., 2002, 159, 1.

34 K. Matyjaszewski and T. P. Davis, Handbook of Radical Polymerization, John Wiley \& Sons Inc., 2002.

35 C. L. McCormick, B. S. Sumerlin, B. S. Lokitz and J. E. Stempka, Soft Matter, 2008, 4, 1760.

36 P. De, S. R. Gondi, D. Roy and B. S. Sumerlin, Macromolecules, 2009, 42, 5614.

37 M. Barz, F. K. Wolf, F. Canal, K. Koynov, M. J. Vicent, H. Frey and R. Zentel, Macromol. Rapid Commun., 2010, 31, 1492.

38 M. L. Becker, J. Liu and K. L. Wooley, Biomacromolecules, 2005, 6, 220.

39 T. Diaz, A. Fischer, A. Jonquieres, A. Brembilla and P. Lochon, Macromolecules, 2003, 36, 2235.

40 R. E. Richard, M. Schwarz, S. Ranade, A. K. Chan, K. Matyjaszewski and B. Sumerlin, ACS Symp. Ser., 2006, 944, 234.

41 X. Jiang, Z. Ge, J. Xu, H. Liu and S. Liu, Biomacromolecules, 2007, 8, 3184.

42 M. Licciardi, Y. Tang, N. C. Billingham, S. P. Armes and A. L. Lewis, Biomacromolecules, 2005, 6, 1085. 
43 V. Buetuen, S. Liu, J. V. M. Weaver, X. Bories-Azeau, Y. Cai and S. P. Armes, React. Funct. Polym., 2006, 66, 157.

44 F. F. Wolf, N. Friedemann and H. Frey, Macromolecules, 2009, 42, 5622.

45 P. A. Wilbon, Y. Zheng, K. Yao and C. Tang, Macromolecules, 2010, 43, 8747.

46 M. Spasova, L. Mespouille, O. Coulembier, D. Paneva, N. Manolova, I. Rashkov and P. Dubois, Biomacromolecules, 2009, 10, 1217.

47 J. Lee, Y. H. Bae, Y. S. Sohn and B. Jeong, Biomacromolecules, 2006, 7, 1729.

48 S. Santra, C. Kaittanis and J. M. Perez, Langmuir, 2010, 26, 5364.
49 L. Zhang, R. Guo, M. Yang, X. Jiang and B. Liu, Adv. Mater., 2007, 19, 2988

50 A. O. Saeed, S. Dey, S. M. Howdle, K. J. Thurecht and C. Alexander, J. Mater. Chem., 2009, 19, 4529.

51 L. Zhang, Y. Hu, X. Jiang, C. Yang, W. Lu and Y. H. Yang, J. Controlled Release, 2004, 96, 135.

52 J. Jiang, B. Qi, M. Lepage and Y. Zhao, Macromolecules, 2007, 40, 790.

53 J. Jiang, X. Tong, D. Morris and Y. Zhao, Macromolecules, 2006, 39, 4633. 